\title{
Brain-Derived Neurotrophic Factor Precursor in the Hippocampus Regulates Both Depressive and Anxiety-Like Behaviors in Rats
}

\begin{abstract}
Feng Zhong ${ }^{1}$, Lei Liu ${ }^{1,2}$, Jia-Li Wei ${ }^{1}$, Zhao-Lan Hu ${ }^{1}$, Li Li ${ }^{1,2}$, Shuang Wang ${ }^{3}$, Jun-Mei $X u^{1,2}$, Xin-Fu Zhou ${ }^{4}$, Chang-Qi Li ${ }^{5}$, Zhao-Yun Yang ${ }^{1,2 *}$ and Ru-Ping Dai ${ }^{1,2 *}$

${ }^{1}$ Department of Anesthesiology, The Second Xiangya Hospital, Central South University, Changsha, China, ${ }^{2}$ Anesthesia Medical Research Center of Central South University, Changsha, China, ${ }^{3}$ Medical Research Center and Clinical Laboratory, Xiangya Hospital of Central South University, Changsha, China, ${ }^{4}$ Division of Health Sciences, School of Pharmacy and Medical Science and Sansom Institute, University of South Australia, Adelaide, SA, Australia, ${ }^{5}$ Department of Anatomy and Neurobiology, School of Basic Medical Science, Central South University, Changsha, China
\end{abstract}

Depression and anxiety are two affective disorders that greatly threaten the mental health of a large population worldwide. Previous studies have shown that brain-derived neurotrophic factor precursor (proBDNF) is involved in the development of depression. However, it is still elusive whether proBDNF is involved in anxiety, and if so, which brain regions of proBDNF regulate these two affective disorders. The present study aims to investigate the role of proBDNF in the hippocampus in the development of depression and anxiety. Rat models of an anxiety-like phenotype and depression-like phenotype were established by complete Freund's adjuvant intra-plantar injection and chronic restraint stress, respectively. Both rat models developed anxiety-like behaviors as determined by the open field test and elevated plus maze test. However, only rats with depression-like phenotype displayed the lower sucrose consumption in the sucrose preference test and a longer immobility time in the forced swimming test. Sholl analysis showed that the dendritic arborization of granule cells in the hippocampus was decreased in rats with depression-like phenotype but was not changed in rats with anxiety-like phenotype. In addition, synaptophysin was downregulated in the rats with depression-like phenotype but upregulated in the rats with anxiety-like phenotype. In both models, proBDNF was greatly increased in the hippocampus. Intra-hippocampal injection anti-proBDNF antibody greatly ameliorated the anxiety-like and depressive behaviors in the rats. These findings suggest that despite some behavioral and morphological differences between depression and anxiety, hippocampal proBDNF is a common mediator to regulate these two mental disorders.

Keywords: proBDNF, depression, anxiety, hippocampus, stress

\section{INTRODUCTION}

Depression and anxiety are highly debilitating mental disorders that severely affect patients' quality of life and put a burden on families and society. Globally, depression ranks as the largest contributor to global disability and nearly 300 million people suffer from anxiety. Around half of them have comorbidity of depression and anxiety $(1,2)$. However, studies on depression and anxiety 
mechanisms and the invention of therapeutic drugs develop slowly. Clinical drugs take weeks to months to have therapeutic effects, while more than one-third of patients are still resistant to the treatment (3). Therefore, it is urgent to explore the underlying mechanism of depression and anxiety.

Brain-derived neurotropic factor (BDNF) is a member of the neurotrophin family of growth factors. It is widely expressed in different brain regions including the amygdala, hippocampus and neocortex (4). It is well known that BDNF exerts antidepressant effects in various experimental models (5-8). Notably, BDNF is first synthesized as the BDNF precursor (proBDNF), which is then intracellularly cleaved by proconvertases/furin or extracellularly processed by matrix metalloproteinases /plasmin to generate mature $\operatorname{BDNF}(9,10)$. Besides being an intermediate during the synthesis of mature BDNF, proBDNF can act on its receptors and have opposite functions to mBDNF in regulating neuronal activity (11). In this regard, BDNF binds to its receptor TrkB to promote neuronal survival, differentiation and synaptic plasticity. In contrast, proBDNF induces neuronal apoptosis via activation of a receptor complex of p75 neurotrophin receptor $\left(\mathrm{p} 75^{\mathrm{NTR}}\right)$ and sortilin $(12,13)$. Furthermore, proBDNF negatively regulates dendritic complexity and depresses the synaptic transmission in the hippocampus (14). Thus, proBDNF in the hippocampus may have different biological functions in anxiety/depression than mature BDNF.

Accumulating evidence has shown that proBDNF signaling is involved in the disease progress of depression. For example, clinical studies have shown that proBDNF was decreased in the postmortem cerebellum and spleen of depressed patients (15). proBDNF and its receptors $\mathrm{p} 75^{\mathrm{NTR}}$ and sortilin were upregulated in the serum of female depressed patients and positively correlated with depression scores $(16,17)$. Furthermore, the increase in proBDNF in the serum of depressed patients was reversed by long-term antidepressant treatment (16). Experimental studies have also shown that proBDNF and its receptors were increased with spine loss in the hippocampus of rats with depression, that has been induced by unpredictable, chronic, mild stress (18). Anti-proBDNF antibody (Ab-proBDNF) injection via intra-cerebroventricular and intraperitoneal approaches reversed the stress-induced depressive behavior (18). Moreover, proBDNF was upregulated in the medial prefrontal cortex while downregulated in the nucleus accumbens of learned helplessness rats $(19,20)$. The role of hippocampal proBDNF on regulating depressive behavior and the exact neuronal target for depression treatment are not yet clear. Moreover, the role of hippocampal proBDNF signaling in anxiety disorder is still unknown. Anxiety disorder, although sharing some symptoms with depression, is a different mental illness (21-23). In particular, anxiety usually precedes depression and eventually develops into depression (24). It was shown that hippocampal proBDNF was increased in carioca high-conditioned freezing rats, an anxiety disorder model (25). However, whether the increased hippocampal proBDNF is involved in anxiety disorders is still unknown.

This study aims to explore the possible role of hippocampal proBDNF in depression and anxiety. The rat model of anxiety was induced by complete Freund's adjuvant (CFA) intra-plantar injection and the depressed rat model was established by chronic restraint stress (CRS). The present study showed that proBDNF was upregulated in the hippocampus in these two animal models. Neutralizing the increased endogenous hippocampal proBDNF by the monoclonal Ab-proBDNF ameliorated the anxiety-like and depressive behaviors. Thus, the present study suggests that the hippocampal proBDNF is a common mediator that regulates depression and anxiety.

\section{MATERIALS AND METHODS}

Male Sprague Dawley rats (eight weeks old; weight, $250 \pm$ $20 \mathrm{~g}$ ) were purchased from Hunan SJA Laboratory Animal Co., Ltd. (Changsha, China). During the experiment, animals were kept in a room with a 12-h light/dark cycle and environmental temperature of $25^{\circ} \mathrm{C}$ with $50-60 \%$ humidity in the experimental animal center of The Second Xiangya Hospital. Rats were housed in standard cages with ad libitum access to food and water. All of the animals were habituated for at least 1 week before any manipulation. All of the procedures were approved by the Institution of Animal Care and Use Committee of The Second Xiangya Hospital and Use Committee, and conformed to the Guide for the Care and Use of Laboratory Animals.

\section{Animal Models}

Depression-like behavior was induced by CRS in our previous study (26). Rats were restricted in a transparent cone made of polyvinyl chloride films. The restraint was performed $4 \mathrm{~h}$ per day for 3 weeks, and animals could not move except for breathing. The time of the restraint was random from 10:00 to 14:00. Then, rats were released from the cone back to the home cage after the restraint. The control rats were handled by the same experimenter without any restraints. Another group of rats was used for CFA (Sigma-Aldrich, St. Louis, MO, United States) injection. First, $100 \mu \mathrm{l} \mathrm{CFA} \mathrm{was} \mathrm{injected} \mathrm{into} \mathrm{the} \mathrm{left} \mathrm{footpad} \mathrm{to}$ induce anxiety-like behavior as described previously (27). Three weeks later, rats were subjected to a battery of behavioral tests for the assessment of nociceptive responses, anxiety-like behaviors and depression-like behaviors.

\section{Nociceptive Behavioral Test}

The mechanical allodynia induced by CFA intra-plantar injection was accessed by measuring the $50 \%$ paw withdrawal threshold (PWT), as described previously $(28,29)$. The $50 \%$ PWT in response to a series of von Frey filaments (Ugo basile, Gemonio, Italy) was determined by the up and down method. Briefly, rats were placed separately in plexiglass chambers with mesh floors for $30 \mathrm{~min}$ to habituate before the test. Eight von Frey filaments with bending forces ranging from 0.6 to $15 \mathrm{~g}$ were chosen. Firstly, the $2.0 \mathrm{~g}$ filament was applied perpendicular to the plantar surface of the paw for every trial. If a positive response (apparent withdrawal, licking, jumping) occurred, an "X" was recorded. Then, a weaker filament was applied. If no positive response occurred, we recorded an "O," and a stronger filament was applied. Each trial ended when a six-number sequence of Os and Xs was obtained. The maximum and minimum limitation for filaments forces was to 0.6 and $15 \mathrm{~g}$. Finally, the 50\% PWT 
was achieved by an adjusted version of the formula presented by Chaplan (28).

\section{Open Field Test}

Animals were placed in the center area of an open arena $(120 \mathrm{~cm}$ long* $120 \mathrm{~cm}$ wide* $40 \mathrm{~cm}$ high) and were free to explore the field for $5 \mathrm{~min}$. All of the movements were tracked by the overhead camera. The total travel distance, the time and travel distance in the central square $(80 \mathrm{~cm}$ wide* $80 \mathrm{~cm}$ long) were analyzed by ViewPoint Video Tracking Software (ViewPiont Behavior Technology, Lyon, France).

\section{Elevated Plus Maze Test}

Animals were placed in the plus maze with a central area $(10 \mathrm{~cm}$ long* $10 \mathrm{~cm}$ wide), two open arms (50 cm long* $10 \mathrm{~cm}$ wide) and two closed arms ( $50 \mathrm{~cm}$ long* $10 \mathrm{~cm}$ wide* $40 \mathrm{~cm}$ high). This maze was $50 \mathrm{~cm}$ above the ground. Animals were free to explore the $4 \mathrm{arms}$ for $5 \mathrm{~min}$. All of the movements were recorded by the overhead camera. The travel time and number of entries into the open/closed arms were analyzed by ViewPoint Video Tracking Software (ViewPiont Behavior Technology, Lyon, France).

\section{Sucrose Preference Test}

Animals were housed individually in cages, and provided with a bottle of water and a bottle of $1 \%$ sucrose solution. The position of the two bottles was switched to reduce the bias for place preference after $24 \mathrm{~h}$. At the end of $48 \mathrm{~h}$, bottles were removed and the liquid consumption was recorded. The percentage of sucrose intake was calculated as sucrose intake/total fluid intake $* 100 \%$.

\section{Forced Swimming Test}

Animals were placed in a plexiglass cylinder $(20 \mathrm{~cm}$ diameter $\times 60 \mathrm{~cm}$ height), filled with $30 \mathrm{~cm}$ of water maintained at $\sim 25$ $\pm 1^{\circ} \mathrm{C}$ and were forced to swim for $15 \mathrm{~min}$ to habituate. The next day, animals were placed in the water again for $5 \mathrm{~min}$, and all of the movements were recorded by the overhead camera. The immobile time was assessed by ViewPoint Video Tracking Software (ViewPiont Behavior Technology, Lyon, France).

\section{Western Blot}

After being deeply anesthetized by sevoflurane, animals were decapitated and hippocampus tissue was collected on ice. Protein lysates were prepared as previously described (29). Then, $50 \mu \mathrm{g}$ protein was loaded and separated by a $15 \%$ SDS-PAGE gel, and then transferred to a PVDF membrane (Millipore, Billerica, MA, United States) at $200 \mathrm{~mA}$ for $2 \mathrm{~h}$. After incubation in $1 \%$ gelatin solution for blocking for $1 \mathrm{~h}$ at room temperature, rabbit anti-BDNF (Santa Cruz Biotechnology Cat\# sc-546, RRID:AB_630940), rabbit anti-p75 (Abcam Cat\# ab8874, RRID:AB_306827), rabbit-anti-sortilin (Abcam Cat\# ab16640, RRID:AB_2192606), mouse anti-MAP2 (Boster Cat\# BM1243), rabbit anti-synaptophysin(SYP) (Proteintech Cat\# 17785-1-AP) and mouse anti-GAPDH (CMCTAG Cat\# AT0002) were applied to the membrane at $4^{\circ} \mathrm{C}$ overnight. Then the membrane was incubated with HRP-conjugated goat anti-rabbit IgG (SigmaAldrich Cat\# A0545, RRID:AB_257896) or goat anti-mouse IgG (Sigma-Aldrich Cat\# A9044, RRID:AB_258431) at room temperature for $1 \mathrm{~h}$. The immunoreactivity of the proteins on the membrane was detected with an enhanced chemiluminescence kit (Millipore Cat\# WBKL S00 50) and x-ray film (Carestream, United States). The band intensity was quantified using Image J software (NIH, Bethesda, MD, United States).

\section{Immunohistochemistry}

After being deeply anesthetized by overdose chloral hydrate (400 mg/kg), animals were cardiac perfused with $100 \mathrm{ml}$ normal saline and followed by $300 \mathrm{ml} 4 \%$ ice-cold paraformaldehyde. The brain was harvested and post-fixed in $4 \%$ paraformaldehyde at $4^{\circ} \mathrm{C}$ overnight. Then the brain was dehydrated in $30 \%$ sucrose in phosphate buffer saline (PBS). The hippocampus region of the brain was sliced in a cryostat (CM1950, Leica Biosystems, Germany). Sections were washed in PBS and rinsed in 3\% $\mathrm{H}_{2} \mathrm{O}_{2}$ for $30 \mathrm{~min}$ to remove endogenous peroxidase. Then, the sections were blocked by $5 \%$ BSA containing $0.01 \%$ Triton X100 in PBS at room temperature for $2 \mathrm{~h}$. Primary antibody antiproBDNF monoclonal antibody which was generated by us and has been characterized previously was applied overnight at $4^{\circ} \mathrm{C}$ $(29,30)$. The sections were incubated in biotinylated goat antimouse immunoglobulin (Jackson ImmunoResearch Labs Cat\# 111-065-003, RRID:AB_2337959) and followed by an ABC kit (Vector Laboratories Cat\# PK-4000, RRID:AB_2336818). The glucose oxidase-DAB-nickel method was used for visualization (31). Finally, all of the sections were transferred onto gelatincoated slides and dehydrated. The slides were cover-slipped with neutral balsam (ZSGB-BIO Cat\# ZLI-9555) for visualization by optical microscopy (BX53, Olympus Corporation, Japan).

For confirmation of the Ab-proBDNF injection site, we performed the immunohistochemistry procedure mentioned above except for the application of the primary antibody.

\section{Golgi Staining}

The staining procedure was carried out using an FD Rapid GolgiStainTM kit (FD Neuro-Technologies Cat\# PK-401). In brief, animals were deeply anesthetized by sevoflurane and decapitated. The brain was collected and rinsed in Golgi-Cox solution, a mixture of solution $\mathrm{A}$ and solution $\mathrm{B}$, and kept for at least 2 weeks in the dark. Then the tissue was kept in solution $\mathrm{C}$ for cryo-protection for 3 days. The brain was sliced into $150 \mu \mathrm{m}$ sections by a cryostat (CM1950, Leica Biosystems, Germany). The hippocampus sections were transferred onto gelatin-coated slides. A mixture of solution D and solution $\mathrm{E}$ was used to visualize the neuronal architecture. After dehydration, the slides were cover-slipped with neutral balsam for visualization by optical microscopy (BX53, Olympus Corporation, Japan). Dendrite branches were traced by Image J software $(\mathrm{NIH}$, Bethesda, MD, United States) with the NeuronJ plugin (32). Then dendritic length and spine density were calculated. Neuronal arborization was analyzed by counting the number of crossings by dendrites of concentric circles originating at the soma with increasing radii of $20 \mathrm{~mm}$, using the sholl analysis plugin in ImageJ (33). 


\section{Stereotaxic Surgery and Drug Infusion}

The surgery was performed according to a standardized protocol $(34,35)$. After induction with $5 \%$ sevoflurane in the anesthesia chamber, rats were placed in a stereotaxic apparatus (68025, RWD Life Science, China) with continuous $2.5 \%$ sevoflurane inhalation. Two stainless steel guide cannulas were implanted bilaterally with the cannula tips $1.5 \mathrm{~mm}$ above the dentate gyrus (DG) area (AP-4.2 mm; ML $\pm 2.5 \mathrm{~mm}$; DV-4.5 mm) (36). The guide cannula was secured with dental cement anchored to the skull. Stylets inserted into each cannula to maintain patency until the rats were subjected to delivery of drugs. The injection needle was inserted into the guide cannula, with its tip located $1.5 \mathrm{~mm}$ beyond the end of the guide cannula. Next, $1 \mu \mathrm{l}$ monoclonal anti-proBDNF (29) antibody $(1 \mu \mathrm{g} / \mu \mathrm{l})$ was injected into the DG area in each hemisphere on day 22. The same volume of IgG $(1 \mu \mathrm{g}, 1 \mu \mathrm{g} / \mu \mathrm{l})$ (CMCTAG Cat\# AT1596) or normal saline was injected bilaterally into the same sites in the control group. The same dose of regents was administered repeatedly on day 28. From day 29 to 34, a series of behavioral tests was performed. Then rats were killed, and the brain was harvested for identification of the injection site or further analysis.

\section{Statistical Analysis}

Data are expressed as mean \pm SEM. Statistical analyses were performed using an unpaired two-tailed Student's $t$-test, oneway analysis of variance or two-way analysis of variance where appropriate. $p$-values were accepted as significantly different at $p<0.05$. The statistical program used was GraphPad Prism 6.0 (San Diego, CA, United States).

\section{RESULTS}

\section{Different Behaviors in the Rats With Anxiety-Like Phenotype and Rats With Depression-Like Phenotype}

Clinically, depression and anxiety result in different behaviors that can be substantiated via various behavioral tests: the selfrating anxiety scale, self-rating depression scale, or Hamilton depression scale tests. In rats, anxiety-like behavior can be induced by persistent inflammatory pain. It has been noticed that CFA intra-plantar injection rendered pain hypersensitivity as indicated by the decreased PWT (Figures 1A,B). Moreover, CRStreated rats did not display mechanical hyperalgesia, whereas locomotor activity was not altered by CRS and CFA injection (Figure 1C). Both rats treated with CFA and those treated with CRS displayed decreased traveled distance in the central square in the OFT (Figure 1D) and reduced time spent in the open arms in the EPM (Figure 1E). In contrast, the SPT results showed that CFA-treated rats had comparable sucrose consumption with the controls whereas CRS-treated rats consumed less sucrose than both the controls and CFA-treated rats (Figure 1F). Similarly, in the FST, the immobility time of CRS-treated rats was longer than that of the controls and CFA-treated rats (Figure 1G). Thus, CRStreated rats displayed anxiety- and depression-like behaviors, whereas CFA -treated rats only displayed anxiety-like behaviors.

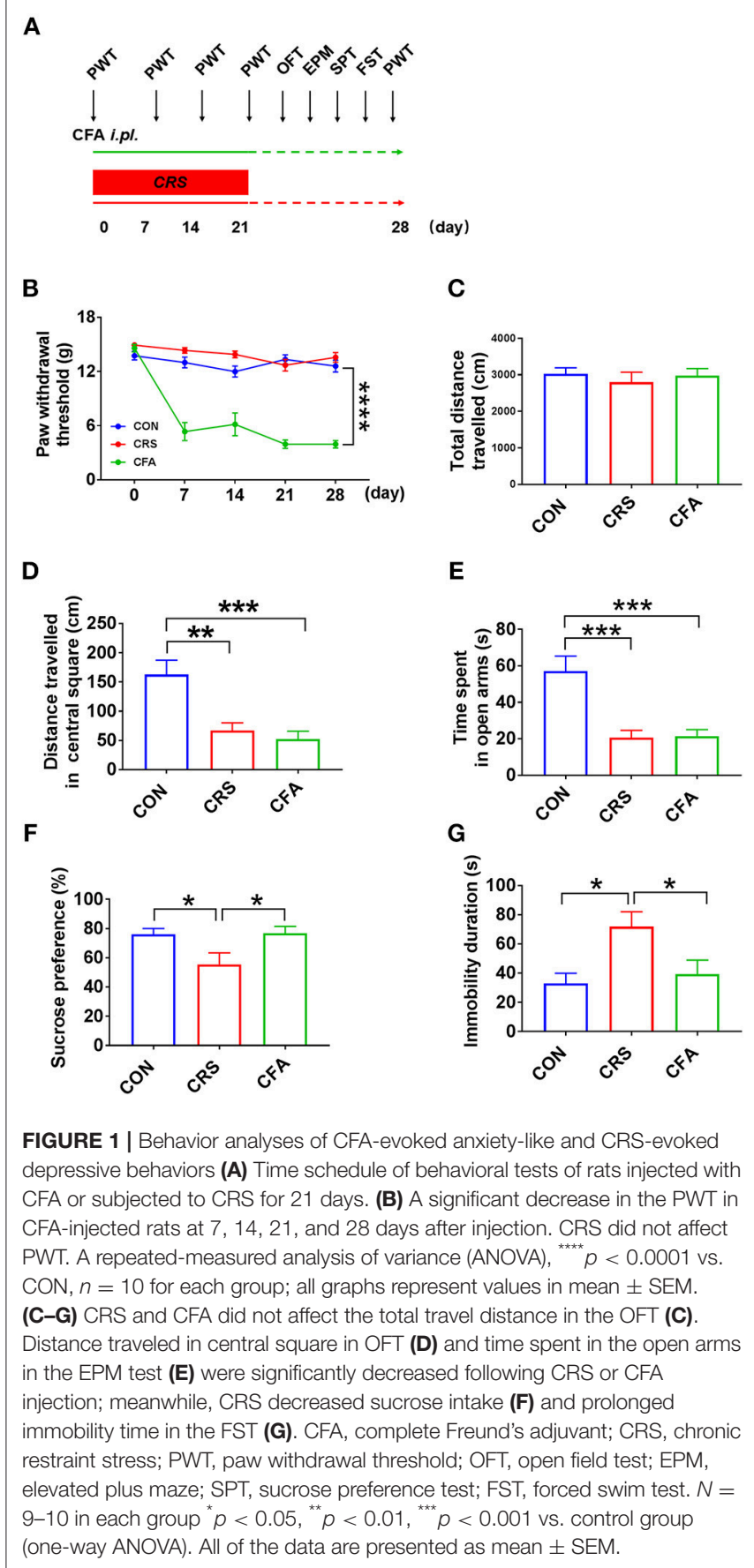

\section{Different Granule Cell Morphology and Synaptic Integrity Changes Between} Anxiety-Like and Depression-Like Behavior It has been reported that depression and anxiety involve synaptic changes (37-39). Therefore, we examined whether there were synaptic changes in these two animal models. Rats with depression-like phenotype and rats with anxiety-like phenotype had similar spine density and dendritic length of granule cells in the DG area compared with the controls 
(Figures 2A,B,D). However, sholl analysis showed a reduced complexity of granule neurons in rats with depression-like phenotype, and there was no difference in the dendritic arborization in rats with anxiety-like phenotype compared with the control group (Figures 2C,E). To assess the effect of CRS and CFA exposure on synaptic integrity, the expression levels of synaptophysin and microtubule-associated protein 2 (MAP2) in the hippocampus were measured. Synaptophysin was downregulated in the rats with depression-like phenotype but upregulated in the rats with anxiety-like phenotype. However, there was no significant change in MAP2 levels in the rats with anxiety-like phenotype and rats with depression-like phenotype as compared with the controls (Figures 2F,G). These results suggest that different morphologies and synaptic changes were responsible for depression and anxiety.
A

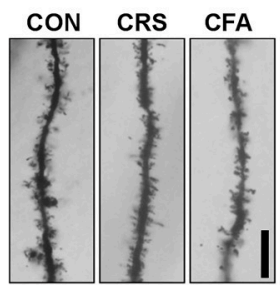

B

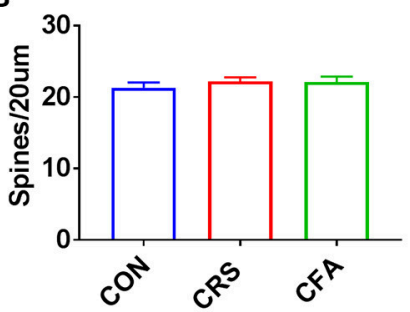

D

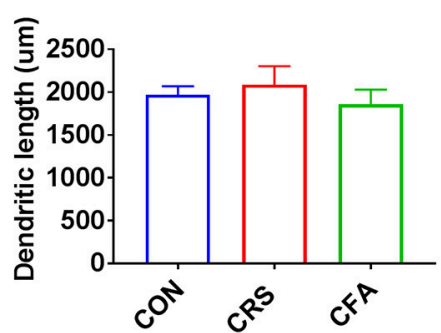

$\mathbf{F}$

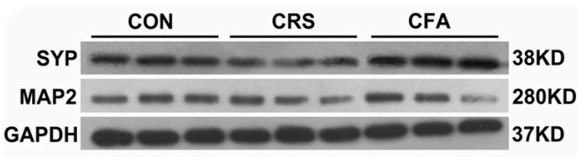

E
C
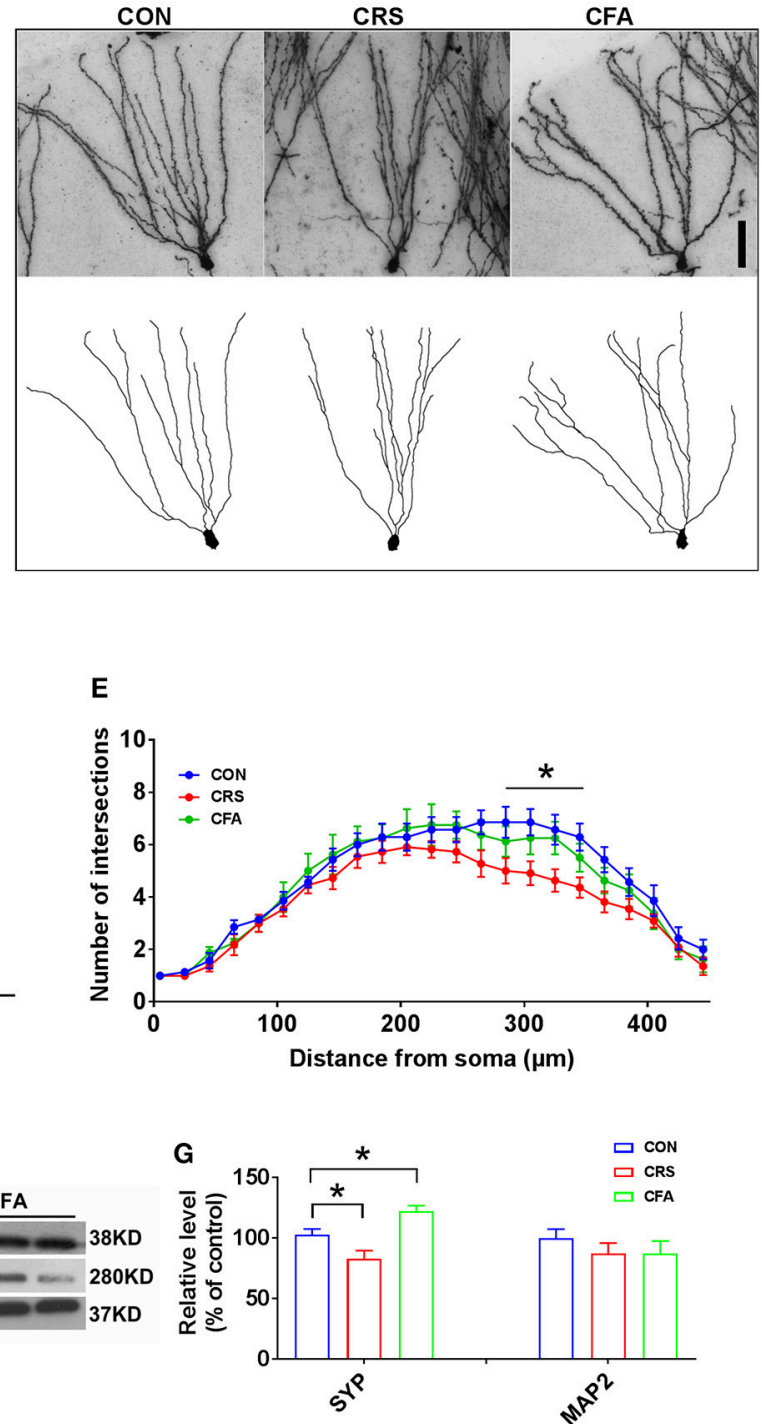

FIGURE 2 | Granule cell morphology and synaptic integrity changes between anxiety-like and depression-like behaviors. (A,B) Representative Golgi-Cox staining images of dendritic spines and spine density in the hippocampal DG granule cells in CRS-treated rats, CFA-treated rats, and the controls. $n=10$ for each group, bar $=10 \mu \mathrm{m}$. (C) Representative images of Golgi-Cox -stained granule cells in the DG area (top) and the reconstruction of its dendritic branches (bottom) from each group. Bar $=50 \mu \mathrm{m}$. (D) There were no significant effects of CRS and CFA injection on the dendritic length of the DG granule cells. (E) Sholl analysis of dendritic length in DG granule cells. CRS reduced dendrite intersection in the region 285-345 $\mathrm{mm}$ away from the soma compared with the control group. A repeated measures analysis of variance (ANOVA), ${ }^{*} p<0.05$ vs. CON, $n=10$ for each group; all graphs represent mean \pm SEM. (F,G) Expression of SYP and MAP2 in the hippocampus was evaluated by Western blotting. Semi-quantitative analyses of SYP and MAP2 expression were performed. Note that the expression of SYP was significantly reduced in CRS-treated rats but was increased in CFA -treated rats. DG: dentate gyrus; CFA: complete Freund's adjuvant; CRS, chronic restraint stress; SYP, synaptophysin; MAP2, microtubule-associated protein $2 .{ }^{*} p<0.05$, vs. control group (one-way ANOVA). All of the data are presented as mean \pm SEM. 


\section{Differential Expression of proBDNF and its Receptors in the Hippocampus in Rats With Anxiety-Like Phenotype and Rats With Depression-Like Phenotype}

A recent study showed that chronic, unpredictable, mild stress upregulates proBDNF in the hippocampus of rats with depressive behavior (18). To further confirm the upregulation of proBDNF in depression and anxiety disorders, we examined the expression of proBDNF and mature BDNF in the hippocampus of these two rat models established in the present study. As shown in Figures $\mathbf{3 A}, \mathbf{B}$, proBDNF was found upregulated in the hippocampus. Interestingly, there was no significant change in BDNF levels in rats with depression-like phenotype or rats with anxiety-like phenotype as compared with the controls. These findings suggest that both depression and anxiety render the upregulation of hippocampal proBDNF. Surprisingly, the expression of hippocampal p $75^{\mathrm{NTR}}$ was not significantly changed in the rats with CRS-evoked depression-like behavior but was greatly down-regulated in the rats with CFA-evoked anxietylike behavior. In addition, there was no significant difference in sortilin expression among the rats with anxiety-like phenotype, the rats with depression-like phenotype and the control group. Furthermore, proBDNF expression was intensely upregulated in the hippocampus of rats with depression-like phenotype and rats with anxiety-like phenotype (Figure 3C). These results suggest that depression and anxiety may have discrepant expression of proBDNF signaling.

\section{Intra-Hippocampal Injection of Ab-proBDNF Attenuated Anxiety-Like and Depressive Behaviors}

The increased proBDNF in the hippocampus in the rats with anxiety-like phenotype and rats with depression-like phenotype may contribute to disease progress. In order to test this hypothesis, bilateral intra-hippocampal injection of monoclonal Ab-proBDNF (1 $\mu \mathrm{g}$ each side) was performed twice in the normal rats through the cannula. As shown in Figures 4A,B, intra-hippocampal injection of Ab-proBDNF was limited within the DG area, suggesting injection precision. Intra-hippocampal injection of Ab-proBDNF in the normal rats did not affect the total travel distance (Figure 4C) and the distance traveled in the central square in the OFT (Figure 4D). The EPM experiment also showed that injection of Ab-proBDNF into the hippocampus did not affect the time spent in the open arms (Figure 4E). These results suggest that intra-hippocampal injection of Ab-proBDNF did not change basal behavior.

As shown in Figure 5A, bilateral intra-hippocampal injection of Ab-proBDNF ( $1 \mu \mathrm{g}$ each side) was performed twice, on day 22 and 28, after CRS or CFA exposure. Intra-hippocampal injection of Ab-proBDNF reversed the reduction of distance traveled in the central area and the decreased time spent in the open
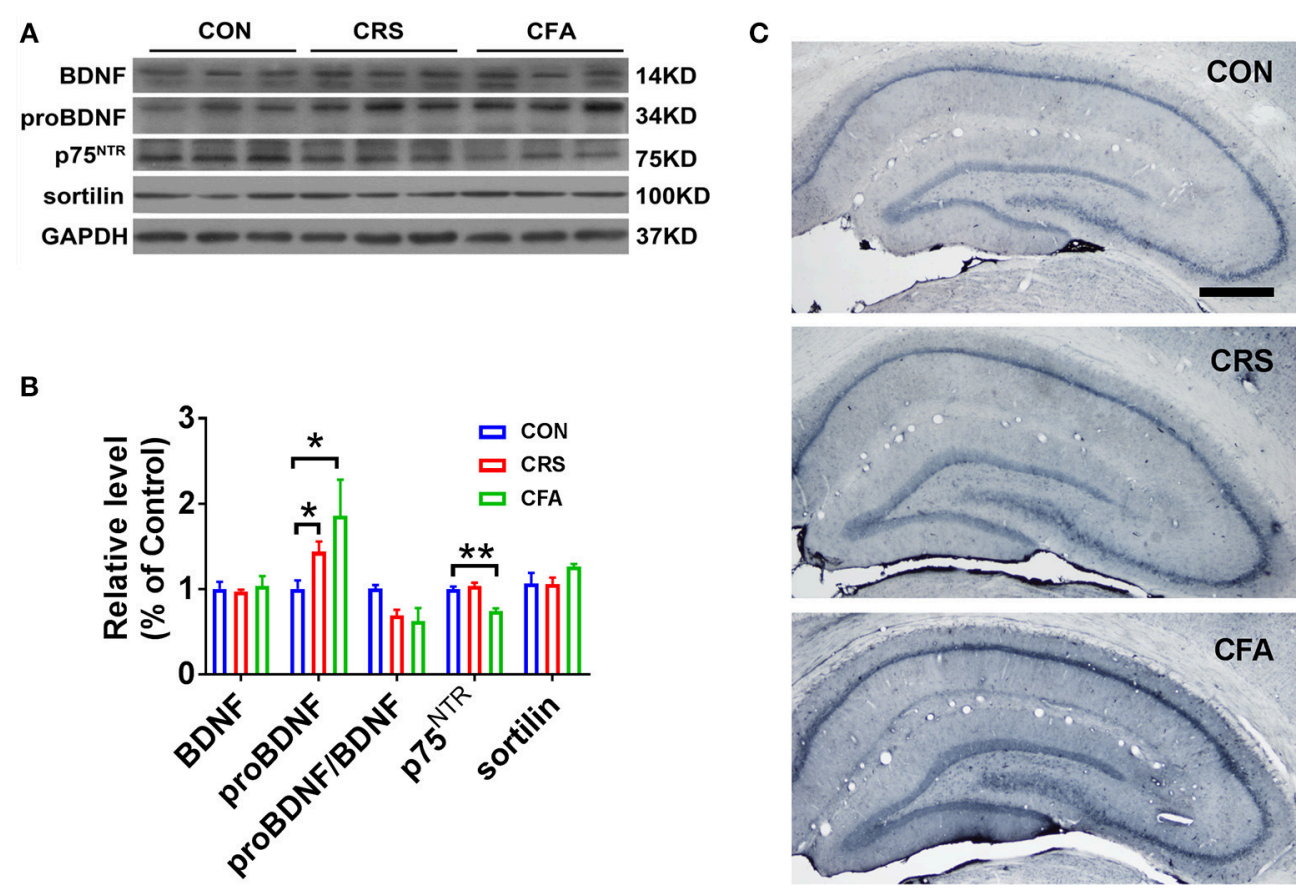

FIGURE 3 | Upregulation of proBDNF and its signaling in rats with anxiety-like phenotype and rats with depression-like phenotype. (A) Representative Western blot, and (B) semi-quantitative analyses of mature BDNF, proBDNF, proBDNF/ BDNF, p75 NTR, and sortilin in the hippocampus of rats with depression-like and anxiety-like phenotype. Note that the expression of proBDNF was increased significantly in the rats with depression-like phenotype and the rats with anxiety-like phenotype. (C) Immunohistochemistry of proBDNF in the hippocampus of rats with depression-like and anxiety-like behaviors. Note that intensive proBDNF was expressed in the hippocampus of the CRS rats and CFA rats. Bar $=100 \mu \mathrm{m}$. ${ }^{*} p<0.05,{ }^{* *} p<0.01$, vs. control group (one-way ANOVA). All of the data are presented as mean \pm SEM. 
A

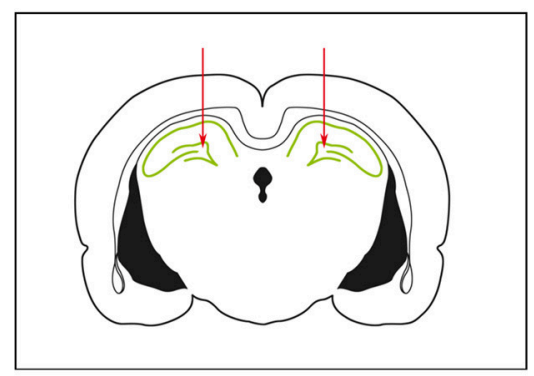

B

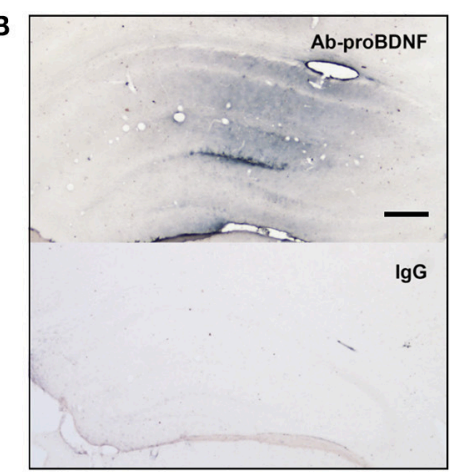

C

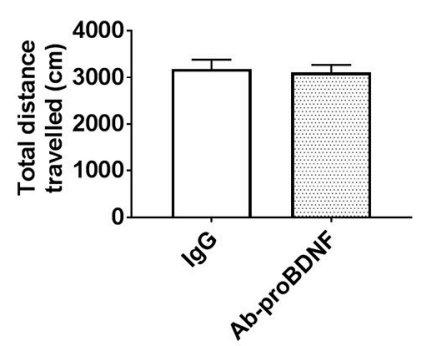

D

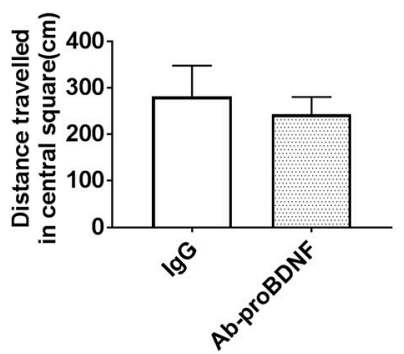

E

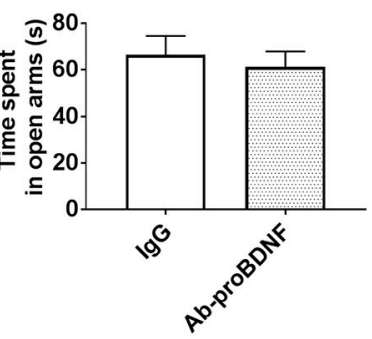

FIGURE 4 | Hippocampal Ab-proBDNF injection and its effect on the anxiety-like behavior in normal rats. (A) Schematic representation of the antibody injection sites into the bilateral DG area through the cannula. (B) Representative immunohistochemistry images of Ab-proBDNF (top) or lgG (bottom) detection by the application of biotinylated anti-mouse IgG and the glucose oxidase-DAB-nickel visualization system 1 week after injection. Bar $=100 \mu \mathrm{m}$. Hippocampal Ab-proBDNF injection did not affect the total travel distance(C) or distance traveled (D) in the central square in the OFT, or the time spent (E) in the open arms in the EPM test. Ab-proBDNF: monoclonal anti-proBDNF antibody. $N=9-10$ in each group. All data are presented as mean $\pm \mathrm{SEM}$.

arms in the rats with anxiety-like phenotype (Figures 5B-D). Similarly, as compared with the vehicle injection group, injection of Ab-proBDNF also inhibited the decreased distance traveled in the central square and the decreased time spent in the open arms in the rats with depression-like phenotype (Figures 5E-G). Moreover, neutralizing the endogenous proBDNF in the hippocampus by Ab-proBDNF injection greatly increased the sucrose consumption in the rats with depression-like phenotype compared with vehicle injection rats (Figure 5H). Finally, FST results showed that the immobility time was significantly lower in the Ab-proBDNF treatment group than in the vehicle treatment group (Figure 5I). Taken together, intra-hippocampal injection of $\mathrm{Ab}$-proBDNF relieved the anxiety-like behavior and exerted an anti-depressive effect.

\section{DISCUSSION}

Anxiety disorders and depression are the two most prevalent mental disorders worldwide (40). In clinical practice, anxiety disorders and depression can overlap; many patients with depression having experienced anxiety disorders earlier in life. Moreover, around $50 \%$ of patients with depression are also diagnosed with an anxiety disorder (2). However, depression also displays different clinical manifestations than anxiety. For example, patients with depression move slowly with flattened or dulled reactions, whereas people with anxiety tend to be more keyed up. In addition, anxiety patients display fear about the future whereas depressed people are less likely to be fraught with worry about future events (41). All of these clinical manifestations suggest that these two distinct mental disorders are closely related, and may share some common mechanisms.

In the experimental rat models, both depressive and anxietylike behaviors displayed the same behaviors in the OFT and EPM tests, which showed a decreased travel distance in the central square of the OFT and a decreased time spent in the open arms of the EPM test. However, depressive behavior can be distinguished from anxiety-like behavior through SPT and FST examinations in which rats with depression-like phenotype had less sucrose consumption and a longer immobility time (42). Like the anxiogenic effect of neuropathic pain $(43,44)$, CFA injection induced persistent inflammatory pain, which developed the anxiety-like behavior 3 weeks after CFA injection. It is consistent with the studies reported previously $(27,45)$. Notably, the chronic inflammatory pain may affect locomotor activity. However, the total traveled distance by rats with anxiety-like behavior in the OFT did not change, thus indicating that the reduction in traveled distance in the central square was not due to the effect of inflammatory pain on locomotor activity. Similarly to results found in previous studies $(26,46-48)$, rats with depression-like phenotype also exhibited lower sucrose consumption in the SPT and a longer immobility time in the FST. These findings further confirmed that there are some overlapping 


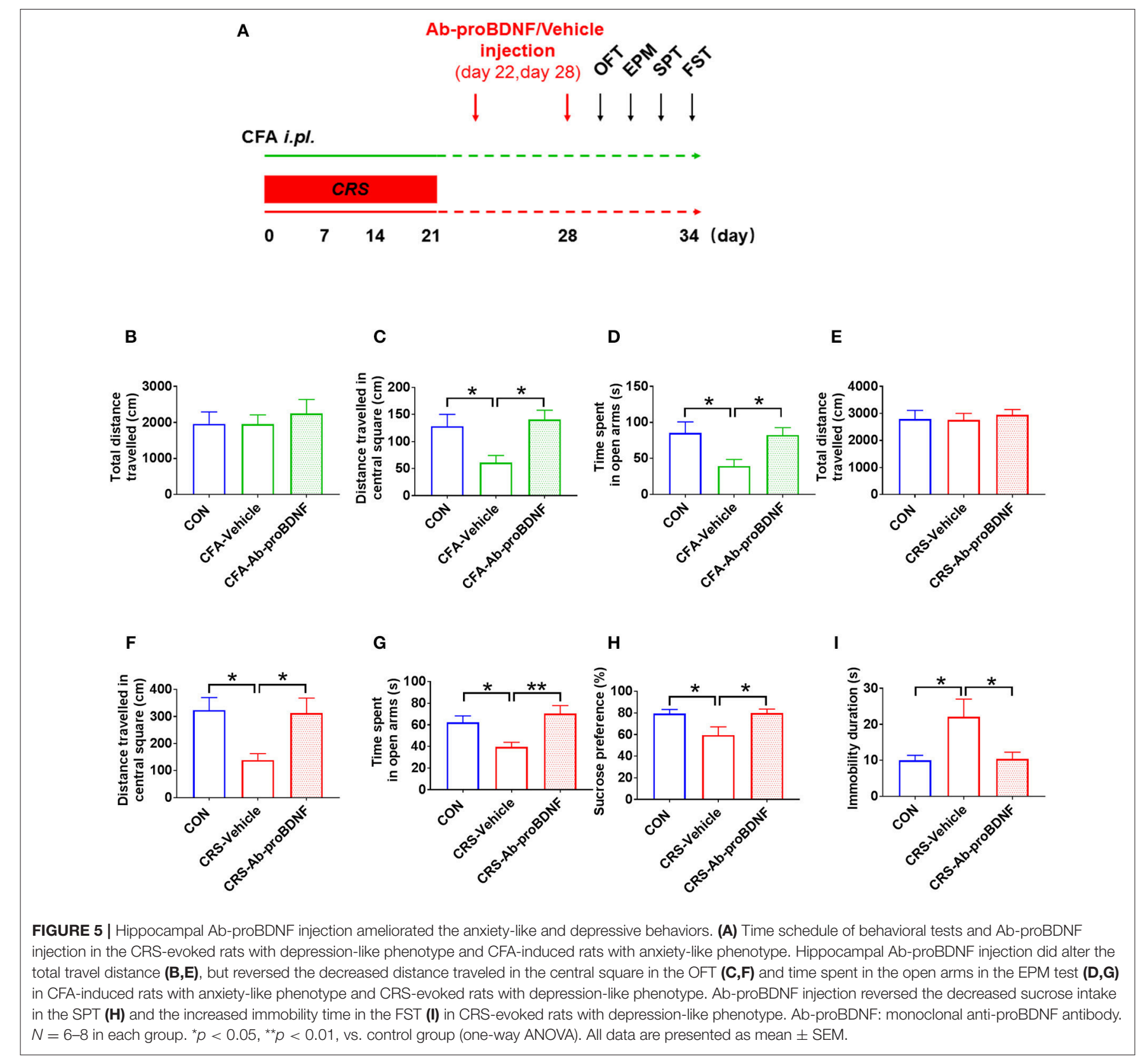

mechanisms between depression and anxiety in experimental animal models; these yet need to be unraveled.

Mental disorders are often accompanied with changes in dendritic arborization and spine density in the neurons of the hippocampus and prefrontal cortex; this is seen in both humans and rodents (49-51). In the present study, the dendritic complexity was significantly decreased in rats with depressionlike phenotype, which is consistent with the results of previous studies (52-54). However, the dendritic length spine density was not changed in the rats with depression-like phenotype, and any difference in dendritic complexity, dendritic length and spine density was not found in the rats with anxiety-like phenotype. Moreover, in the rats with anxiety-like phenotype, synaptophysin, a marker for synaptic density found in the hippocampus, was increased, which is supported by previous reports $(55,56)$. In contrast, hippocampal SYP was decreased in the rats with depression-like phenotype. Whereas, there was no difference found in hippocampal MAP2 among all three groups. These findings suggest that the CRS results in hippocampal dendritic retraction and CFA slightly stimulates synaptogenesis in the hippocampus, providing the neurobiological substrates responsible for the different behaviors related to depression and anxiety.

The precursor of $\mathrm{BDNF}$, proBDNF has been reported to regulate depressive behavior under chronic stress (18). proBDNF was upregulated in the hippocampus, neocortex and medial 
prefrontal cortex in rats with depression-like phenotype. In contrast, the expression of proBDNF was decreased in the nucleus accumbens in the rats with learned helplessness $(19,20)$, suggesting different expression patterns of proBDNF in different brain regions in the depression models. In the current study, the expression of proBDNF was also increased in the hippocampus of rats with depression-like phenotype. Furthermore, the expression of proBDNF signaling was also upregulated in rats with anxietylike phenotype. Although intra-cerebroventricular injection of $\mathrm{Ab}$-proBDNF could attenuate the depressive behavior (18), it is still unclear whether neutralization of the hippocampal proBDNF inhibits the anxiety-like and depressive behaviors.

In the present study, intra-DG injection of Ab-proBDNF in the control rats did not affect behavior as compared with the IgG control treatment, thus suggesting that the reduction of endogenous proBDNF in the hippocampus does not affect the behaviors under physiological conditions. This may be due to the low extracellular level of proBDNF, because only Ab-proBDNF can neutralize the extracellular proBDNF. In contrast to the lack of effect of Ab-proBDNF treatment on behaviors in control rats, Ab-proBDNF injection greatly inhibited the decreased sucrose consumption and increased immobility time in the FST. This indicates that hippocampal proBDNF contributes to the development of depression. Moreover, the intra-hippocampal $\mathrm{Ab}$-proBDNF antibody also greatly protected against the anxietylike behavior, as indicated by the EPM and OFT in both animal models. Collectively, these results indicate that hippocampal proBDNF is a common substrate that regulates depression and anxiety. Notably, in the present study, the injection site of AbproBDNF is mainly limited to the DG region. Previous studies have reported different alterations in the BDNF and proBDNF in CA1, CA3, and DG regions of the hippocampus within rodents with depression-like phenotype (20). Therefore, it would be greatly interesting to explore the role of proBDNF in CA1 and $\mathrm{CA} 3$ regions in depression and anxiety in a future study.

proBDNF exerts its biological effects through binding to its high affinity receptor $\mathrm{p} 75^{\mathrm{NTR}}$ and co-receptor sortilin. It has been reported that $\mathrm{p} 75^{\mathrm{NTR}}$ expression was increased in the rats depressed by unpredictable, chronic, mild stress (18). However, in the rats with CRS-evoked depression-like behavior, the expression of $\mathrm{p} 75^{\mathrm{NTR}}$ was not altered. This contrast may be due to the different paradigms used to induce depression.

\section{REFERENCES}

1. Judd LL, Kessler RC, Pauius MP, Zeller PV, Wittchen HU, Kunovac JL. Comorbidity as a fundamental feature of generalized anxiety disorders: results from the National Comorbidity Study (NCS). Acta Psychiatr Scand. (1998) 98:6-11. doi: 10.1111/j.1600-0447.1998.tb05960.x

2. World Health Organization. Depression and Other Common Mental Disorders: Global Health Estimates. Geneva: World Health Organization (2017).

3. Trivedi MH, Rush AJ, Wisniewski SR, Nierenberg AA, Warden D, Ritz L, et al. Evaluation of outcomes with citalopram for depression using measurementbased care in STAR* D: implications for clinical practice. Am J Psychiatry (2006) 163:28-40. doi: 10.1176/appi.ajp.163.1.28

4. Conner JM, Lauterborn JC, Yan Q, Gall CM, Varon S. Distribution of brain-derived neurotrophic factor (BDNF) protein and mRNA in
Nonetheless, the expression of $\mathrm{p} 75^{\mathrm{NTR}}$ was down-regulated in the rats with anxiety-like phenotype. This is consistent with the finding of a previous study, which showed that the deletion of p $75^{\mathrm{NTR}}$ resulted in anxiety-like behavior (57). In addition, there was no significant difference in sortilin expression in the rats with anxiety-like phenotype compared with the control rats. Recent studies showed that sortilin-knockout mice displayed anxietylike behavior, suggesting the involvement of sortilin in anxiety (58). As there was no significant change in sortilin in the rats with anxiety-like phenotype in the present study, this indicates that sortilin may not contribute to the development of anxiety induced by chronic pain.

In conclusion, the present study showed that depression and anxiety have both distinct and overlapping behaviors, and morphological hippocampal changes in rat models. Furthermore, the increased hippocampal proBDNF played an important role in regulating both depression and anxiety. Inhibition of the increased proBDNF by antibodies might be a potential therapy to treat depression and anxiety.

\section{AUTHOR CONTRIBUTIONS}

FZ: conducted the study, contributed to data collection, data analysis, and manuscript preparation and revision. LeL and J-LW: contributed to the conduction of the study, data collection, and data analysis. Z-LH and SW: helped collect and analyze the data, and revise the manuscript. LiL and J-MX: helped design the study and analyze the data. X-FZ and C-QL: contributed to the experimental design and revision of the manuscript. R-PD and Z-YY: designed and interpreted the work, contributed to data collection, and data analysis, and assisted with manuscript drafting and revision.

\section{ACKNOWLEDGMENTS}

This research was supported by the National Natural Science Foundation of China (81471106 and 81771354 to R-PD), research funding from Health and Family Planning Commission of Hunan Province (132015-028 to Z-YY) and the Hunan Province Science Foundation for Young Scientists of China (2018JJ3864 to SW). We thank Letpub (www.letpub.com) for its linguistic assistance during the preparation of this manuscript. the normal adult rat CNS: evidence for anterograde axonal transport. J Neurosci. (1997) 17:2295-313. doi: 10.1523/JNEUROSCI.17-07-0229 5.1997

5. Scharfman H, Goodman J, Macleod A, Phani S, Antonelli C, Croll S. Increased neurogenesis and the ectopic granule cells after intrahippocampal BDNF infusion in adult rats. Exp Neurol. (2005) 192:348-56. doi: 10.1016/j.expneurol.2004.11.016

6. Shirayama Y, Chen AC, Nakagawa S, Russell DS, Duman RS. Brain-derived neurotrophic factor produces antidepressant effects in behavioral models of depression. J Neurosci. (2002) 22:3251-61. doi: 10.1523/JNEUROSCI.22-08-03251.2002

7. Siuciak JA, Lewis DR, Wiegand SJ, Lindsay RM. Antidepressant-like effect of brain-derived neurotrophic factor (BDNF). Pharmacol Biochem Behav. (1997) 56:131-7. doi: 10.1016/S0091-3057(96)00169-4 
8. Schmidt HD, Duman RS. Peripheral BDNF produces antidepressant-like effects in cellular and behavioral models. Neuropsychopharmacology (2010) 35:2378-91. doi: 10.1038/npp.2010.114

9. Yang J, Siao CJ, Nagappan G, Marinic T, Jing D, Mcgrath K, et al. Neuronal release of proBDNF. Nat Neurosci. (2009) 12:113-5. doi: 10.1038/nn.2244

10. Nagappan G, Zaitsev E, Senatorov VV, Yang J, Hempstead BL, Lu B, et al. Control of extracellular cleavage of ProBDNF by high frequency neuronal activity. Proc Natl Acad Sci USA. (2009) 106:1267-72. doi: 10.1073/pnas.0807322106

11. Martinowich K, Manji H, Lu B. New insights into BDNF function in depression and anxiety. Nat Neurosci. (2007) 10:1089-93. doi: 10.1038/nn1971

12. Lee R, Kermani P, Teng KK, Hempstead BL. Regulation of cell survival by secreted proneurotrophins. Science (2001) 294:1945-8. doi: 10.1126/science.1065057

13. Teng HK, Teng KK, Lee R, Wright S, Tevar S, Almeida RD, et al. ProBDNF induces neuronal apoptosis via activation of a receptor complex of $75^{\mathrm{NTR}}$ and sortilin. $J$ Neurosci. (2005) 25:5455-63. doi: 10.1523/JNEUROSCI.5123-04.2005

14. Yang J, Harte-Hargrove L, Siao CJ, Marinic T, Clarke R, Ma Q, et al. proBDNF negatively regulates neuronal remodeling, synaptic transmission, and synaptic plasticity in hippocampus. Cell Rep. (2014) 7:796-806. doi: 10.1016/j.celrep.2014.03.040

15. Yang B, Ren Q, Zhang JC, Chen QX, Hashimoto K. Altered expression of BDNF, BDNF pro-peptide and their precursor proBDNF in brain and liver tissues from psychiatric disorders: rethinking the brain-liver axis. Transl Psychiatry (2017) 7:e1128. doi: 10.1038/tp.2017.95

16. Jiang H, Chen S, Li C, Lu N, Yue Y, Yin Y, et al. The serum protein levels of the tPA-BDNF pathway are implicated in depression and antidepressant treatment. Transl Psychiatry (2017) 7:e1079. doi: 10.1038/tp.2017.43

17. Zhou L, Xiong J, Lim Y, Ye R, Huang C, Zhu Y, et al. Upregulation of blood proBDNF and its receptors in major depression. J Affect Disord. (2013) 150:776-84. doi: 10.1016/j.jad.2013.03.002

18. Bai YY, Ruan CS, Yang CR, Li JY, Kang ZL, Zhou L, et al. ProBDNF signalling regulates depression-like behaviours in rodents under chronic stress. Neuropsychopharmacology (2016) 41: 2882-92. doi: 10.1038/npp.2 016.100 .

19. Shirayama Y, Yang C, Zhang J-c, Ren Q, Yao W, Hashimoto K. Alterations in brain-derived neurotrophic factor (BDNF) and its precursor proBDNF in the brain regions of a learned helplessness rat model and the antidepressant effects of a TrkB agonist and antagonist. Eur Neuropsychopharmacol. (2015) 25:2449-58. doi: 10.1016/j.euroneuro.2015.09.002

20. Yang B, Yang C, Ren Q, Zhang J-c, Chen Q-X, Shirayama Y, et al. Regional differences in the expression of brain-derived neurotrophic factor (BDNF) pro-peptide, proBDNF and preproBDNF in the brain confer stress resilience. Eur Arch Psychiatry Clin Neurosci. (2016) 266:765-9. doi: 10.1007/s00406-016-0693-6

21. Zimmerman M, Chelminski I. Generalized anxiety disorder in patients with major depression: is DSM-IV's hierarchy correct? Am J Psychiatry (2003) 160:504-12. doi: 10.1176/appi.ajp.160.3.504

22. Eysenck MW, Fajkowska M. Anxiety and depression: toward overlapping and distinctive features. Cogn Emot. (2017) 32:1391-400. doi: 10.1080/02699931.2017.1330255.

23. Zbozinek TD, Rose RD, Wolitzky-Taylor KB, Sherbourne C, Sullivan G, Stein MB, et al. Diagnostic overlap of generalized anxiety disorder and major depressive disorder in a primary care sample. Depress Anxiety (2012) 29:1065-71. doi: 10.1002/da.22026

24. Moffitt TE, Harrington H, Caspi A, Kim-Cohen J, Goldberg D, Gregory AM, et al. Depression and generalized anxiety disorder: cumulative and sequential comorbidity in a birth cohort followed prospectively to age 32 years. Arch Gen Psychiatry (2007) 64:651-60. doi: 10.1001/archpsyc.64.6.651

25. Gisele Pereira D, Renata Lopes F, Lítia Alves DC, Graham C, Danielle B, Lucas Costa H, et al. Hippocampal biomarkers of fear memory in an animal model of generalized anxiety disorder. Behav Brain Res. (2014) 263:34-45. doi: 10.1016/j.bbr.2014.01.012

26. Luo YW, Xu Y, Cao WY, Zhong XL, Duan J, Wang XQ, et al. Insulin-like growth factor 2 mitigates depressive behavior in a rat model of chronic stress. Neuropharmacology (2015) 89:318-24. doi: 10.1016/j.neuropharm.2014.10.011
27. Parent AJ, Beaudet N, Beaudry H, Bergeron J, Bérubé P, Drolet G, et al. Increased anxiety-like behaviors in rats experiencing chronic inflammatory pain. Behav Brain Res. (2012) 229:160-7. doi: 10.1016/j.bbr.2012.01.001

28. Chaplan S, Bach F, Pogrel J, Chung J, Yaksh T. Quantitative assessment of tactile allodynia in the rat paw. J Neurosci Methods (1994) 53:55-63. doi: 10.1016/0165-0270(94)90144-9

29. Luo C, Zhong XL, Zhou FH, Li JY, Zhou P, Xu JM, et al. Peripheral brain derived neurotrophic factor precursor regulates pain as an inflammatory mediator. Sci Rep. (2016) 6:27171. doi: 10.1038/srep27171

30. Li C, Wang H, Ruping D, Cai X, Zhou X. Use of Binding Molecule Specifically Binding to Precursor of Brain-Derived Neurotrophic Factor. U.S. Patent Application No. 15/627,305 (2017).

31. Shu S, Ju G, Fan L. The glucose oxidase-DAB-nickel method in peroxidase histochemistry of the nervous system. Neurosci Lett. (1988) 85:169-71. doi: 10.1016/0304-3940(88)90346-1

32. Meijering E, Jacob M, Sarria JC, Steiner P, Hirling H, Unser M. Design and validation of a tool for neurite tracing and analysis in fluorescence microscopy images. Cytometry A (2004) 58:167-76. doi: 10.1002/cyto.a.20022

33. Ferreira TA, Blackman AV, Oyrer J, Jayabal S, Chung AJ, Watt AJ, et al. Neuronal morphometry directly from bitmap images. Nat Methods (2014) 11:982-4. doi: 10.1038/nmeth.3125

34. Piray A, Daniela H, Patrizia C, Gustav S, James L M, Benno R. Glucocorticoids interact with the hippocampal endocannabinoid system in impairing retrieval of contextual fear memory. Proc Natl Acad Sci USA. (2012) 109:3504-9. doi: 10.1073/pnas. 1200742109

35. Fornari RV, Wichmann R, Atsak P, Atucha E, Barsegyan A, Beldjoud H, et al. Rodent stereotaxic surgery and animal welfare outcome improvements for behavioral neuroscience. J Vis Exp. (2012) 59:e3528. doi: 10.3791/3528

36. Watson C. Paxinos and Watson's The Rat Brain in Stereotaxic Coordinates, 7th Edn. Cambridge: Academic Press (2014).

37. McLaughlin KJ, Gomez JL, Baran SE, Conrad CD. The effects of chronic stress on hippocampal morphology and function: an evaluation of chronic restraint paradigms. Brain Res. (2007) 1161:56-64. doi: 10.1016/j.brainres.2007.05.042

38. Soetanto A, Wilson RS, Talbot K, Un A, Schneider JA, Sobiesk M, et al. Association of anxiety and depression with microtubule-associated protein 2-and synaptopodin-immunolabeled dendrite and spine densities in hippocampal CA3 of older humans. Arch Gen Psychiatry (2010) 67:448-57. doi: 10.1001/archgenpsychiatry.2010.48

39. Alves ND, Correia JS, Patrício P, Mateuspinheiro A, Machadosantos AR, Loureirocampos E, et al. Adult hippocampal neuroplasticity triggers susceptibility to recurrent depression. Transl Psychiatry (2017) 7:e1058. doi: 10.1038/tp.2017.29

40. Kessler RC, Berglund P, Demler O, Jin R, Merikangas KR, Walters EE. Lifetime prevalence and age-of-onset distributions of DSM-IV disorders in the National Comorbidity Survey Replication. Arch Gen Psychiatry (2005) 62:593-602. doi: 10.1001/archpsyc.62.6.593

41. Kendall PC, Watson DE. Anxiety and Depression: Distinctive and Overlapping Features. Cambridge: Academic Press (1989).

42. El Yacoubi M, Bouali S, Popa D, Naudon L, Leroux-Nicollet I, Hamon M, et al. Behavioral, neurochemical, and electrophysiological characterization of a genetic mouse model of depression. Proc Natl Acad Sci USA. (2003) 100:6227-32. doi: 10.1073/pnas.1034823100

43. Roeska K, Doods H, Arndt K, Treede R-D, Ceci A. Anxiety-like behaviour in rats with mononeuropathy is reduced by the analgesic drugs morphine and gabapentin. Pain (2008) 139:349-57. doi: 10.1016/j.pain.2008.05.003

44. Narita M, Kaneko C, Miyoshi K, Nagumo Y, Kuzumaki N, Nakajima M, et al. Chronic pain induces anxiety with concomitant changes in opioidergic function in the amygdala. Neuropsychopharmacology (2006) 31:739-50. doi: 10.1038/sj.npp.1300858

45. Wu Y, Yao X, Jiang Y, He X, Shao X, Du J, et al. Pain aversion and anxiety-like behavior occur at different times during the course of chronic inflammatory pain in rats. J Pain Res. (2017) 10:2585-93. doi: 10.2147/JPR.S139679

46. Naert G, Ixart G, Maurice T, Tapia-Arancibia L, Givalois L. Brain-derived neurotrophic factor and hypothalamic-pituitary-adrenal axis adaptation processes in a depressive-like state induced by chronic restraint stress. Mol Cell Neurosci. (2011) 46:55-66. doi: 10.1016/j.mcn.2010.08.006

47. Chiba S, Numakawa T, Ninomiya M, Richards MC, Wakabayashi C, Kunugi H. Chronic restraint stress causes anxiety-and depression-like 
behaviors, downregulates glucocorticoid receptor expression, and attenuates glutamate release induced by brain-derived neurotrophic factor in the prefrontal cortex. Prog Neuropsychopharmacol Biol Psychiatry (2012) 39:1129. doi: 10.1016/j.pnpbp.2012.05.018

48. Ferraz AC, Delattre AM, Almendra RG, Sonagli M, Borges C, Araujo $\mathrm{P}$, et al. Chronic $\omega-3$ fatty acids supplementation promotes beneficial effects on anxiety, cognitive and depressive-like behaviors in rats subjected to a restraint stress protocol. Behav Brain Res. (2011) 219:116-22. doi: $10.1016 /$ j.bbr.2010.12.028

49. Rosoklija G, Toomayan G, Ellis SP, Keilp J, Mann JJ, Latov N, et al. Structural abnormalities of subicular dendrites in subjects with schizophrenia and mood disorders: preliminary findings. Arch Gen Psychiatry (2000) 57:349-56. doi: 10.1001/archpsyc.57.4.349

50. Goldwater DS, Pavlides C, Hunter RG, Bloss EB, Hof PR, Mcewen BS, et al. Structural and functional alterations to rat medial prefrontal cortex following chronic restraint stress and recovery. Neuroscience (2009) 164:798808. doi: 10.1016/j.neuroscience.2009.08.053

51. Orlowski D, Elfving B, Müller HK, Wegener G, Bjarkam CR. Wistar rats subjected to chronic restraint stress display increased hippocampal spine density paralleled by increased expression levels of synaptic scaffolding proteins. Stress (2012) 15:514-23. doi: 10.3109/10253890.2011.643516

52. Mcewen BS, Nasca C, Gray JD. Stress effects on neuronal structure: hippocampus, amygdala, and prefrontal cortex. Neuropsychopharmacology (2016) 41:3-23. doi: 10.1038/npp.2015.171

53. Christian KM, Miracle AD, Wellman CL, Nakazawa K. Chronic stressinduced hippocampal dendritic retraction requires CA3 NMDA receptors. Neuroscience (2011) 174:26-36. doi: 10.1016/j.neuroscience.2010.11.033

54. McCall T, Weil ZM, Nacher J, Bloss EB, El Maarouf A, Rutishauser $\mathrm{U}$, et al. Depletion of polysialic acid from neural cell adhesion molecule (PSA-NCAM) increases CA3 dendritic arborization and increases vulnerability to excitotoxicity. Exp Neurol. (2013) 241:5-12. doi: 10.1016/j.expneurol.2012.11.028
55. Zhuang F, Mei L, Xin G, Yun W, Wang D, Xing M, et al. The antidepressantlike effect of alarin is related to TrkB-mTOR signaling and synaptic plasticity. Behav Brain Res. (2016) 313:158-71. doi: 10.1016/j.bbr.2016. 06.057

56. Arcego DM, Toniazzo AP, Krolow R, Lampert C, Berlitz C, Garcia EDS, et al. Impact of high-fat diet and early stress on depressive-like behavior and hippocampal plasticity in adult male rats. Molecular Neurobiology (2017) 55:1-14. doi: 10.1007/s12035-017-0538-y

57. Martinowich K, Schloesser RJ, Lu Y, Jimenez DV, Paredes D, Greene JS, et al. Roles of $\mathrm{p} 75^{\mathrm{NTR}}$, Long-term depression, and cholinergic transmission in anxiety and acute stress coping. Biol Psychiatry (2012) 71:75-83. doi: 10.1016/j.biopsych.2011.08.014

58. Ruan CS, Yang CR, Li JY, Luo HY, Bobrovskaya L, Zhou XF. Mice with Sort1 deficiency display normal cognition but elevated anxiety-like behavior. Exp Neurol. (2016) 281:99-108. doi: 10.1016/j.expneurol.2016. 04.015

Conflict of Interest Statement: The authors declare that the research was conducted in the absence of any commercial or financial relationships that could be construed as a potential conflict of interest.

The handling editor declared a shared affiliation, though no other collaboration, with several of the authors FZ, LeL, J-LW, Z-LH, LiL, SW, J-MX, C-QL, Z-YY, and $\mathrm{R}-\mathrm{PD}$, at the time of the review.

Copyright (c) 2019 Zhong, Liu, Wei, Hu, Li, Wang, Xu, Zhou, Li, Yang and Dai. This is an open-access article distributed under the terms of the Creative Commons Attribution License (CC BY). The use, distribution or reproduction in other forums is permitted, provided the original author(s) and the copyright owner(s) are credited and that the original publication in this journal is cited, in accordance with accepted academic practice. No use, distribution or reproduction is permitted which does not comply with these terms. 